\title{
Management of a dental implant displaced into the mandibular bone marrow space: Case reports
}

\author{
Eun Joo Choi ${ }^{1,2}$, Hyo Joon $\mathrm{Kim}^{3}$, and Seong Yong Moon ${ }^{3 *}$ \\ ${ }^{1}$ Department of Oral and Maxillofacial Surgery, Dental Research Institute, Wonkwang University College of Dentistry, Iksan, Republic of Korea \\ ${ }^{2}$ Artificial Intelligence Research Institute of Society, Wonkwang University, Iksan, Republic of Korea \\ ${ }^{3}$ Department of Oral and Maxillofacial Surgery, School of Dentistry, Chosun University, Gwangju, Republic of Korea
}

When an implant fixture is displaced to the bone marrow defect site of the mandible, damage to the inferior alveolar nerve may occur. To minimize postoperative side effects, an accurate radiological evaluation should be made and the best method of removal should be considered. Here, we report two cases of implant removal in patients referred to our hospital who had an implant fixture displaced by a mandibular bone marrow defect, and we also propose a standardized method for removal of the implant. Two patients were referred to our clinic with displacement of an implant fixture to the mandibular bone marrow space. For removal of the implant, a trapezoidal bone window was designed with the buccal cortical bone of the drilling hole as the superior margin. An osteotomy was performed on the cortical bone. The implant was gently moved upwards and removed. Healing was uneventful with no neurological complications.

Key Words: Alveolar bone grafting, Cancellous bone, Dental implant, Implant displacement, Infeior alveolar nerve injury

(c) This is an open-access article distributed under the terms of the Creative Commons Attribution Non-Commercial License (http://creativecommons.org/licenses/by-nc/4.0) which permits unrestricted noncommercial use, distribution, and reproduction in any medium, provided the original work is properly cited.

\section{INTRODUCTION}

Since the first introduction of the osseointegrated implant by Brånemark [1], implants have been widely used as restoration method of missing tooth. Restoration of missing teeth using implants has shown a high success rate. However, as more surgeries progress, various complications have also been reported [2]. The main complications that may occur during implant surgery may be due to the carelessness of the operator or lack of preoperative radiological evaluation. Inadvertent placement of implant may cause damage to the surrounding anatomical structures, such as the root of the adjacent teeth, the inferior alveolar nerve. in severe cases, it will completely pass through the cortical bone and appear to be displaced into the surrounding structure.

In the case of maxillary displacement, the implant fixture may be displaced into the maxillary sinus at the time of implant placement. There have been enough reports of case reports, causes, and removal methods for implants displaced into the maxillary sinus [3,4]. As a method for removing an implant completely displaced into the maxillary sinus, it is standard to remove it through the formation of an additional bony window into the maxillary sinus. If the displaced implant moves to the surrounding paranasal

Received November 11, 2019; Accepted November 21, 2019

*Corresponding author: Seong Yong Moon, Department of Oral and Maxillofacial Surgery, School of Dentistry, Chosun University, 303 Pilmundaero, Dong-gu, Gwangju 61452, Republic of Korea.

Tel: +82-62-220-3810, Fax: +82-62-224-9172, E-mail: msygood@chosun.ac.kr

Copyright $\odot$ 2019, Oral Biology Research Institute 
sinus and is difficult to secure the field of vision by intraoral access, the removal method through functional endoscopic sinus surgery is mainly used [4].

In the case of mandibular displacement, there is a displacement to the bone marrow defect of mandibular body though it is not common [5-15]. If the implant fixture is displaced to the bone marrow defect site of the mandible, damage to the Inferior alveolar nerve may occur. Therefore, in order to minimize possible postoperative side effects, accurate radiological evaluation should be made and the best method of removal should be selected [13]. However, only a few papers report cases of mandibular displaced implants and their treatment.

In this paper, we report a case of implant removal in patients referred to our hospital with implant fixture displaced by a mandibular bone marrow defect, and proposes a standardized removal method of the implant.

\section{Case}

\section{Case 1}

A 72-year-old female patient was referred to Department of Oral and Maxillofacial Surgery due to the displacement of the implant fixture of left second molar region to the mandibular bone marrow space. Her medical history was unremarkable (American Society of Anesthesiologists class I). A panoramic radiograph showed that the fixture (4.0 mm wide, $10.0 \mathrm{~mm}$ long) was displaced inferiorly, approximately $12 \mathrm{~mm}$ below the alveolar ridge (Fig. 1). In the cone beam computed tomography (CBCT) image, the inferior alveolar nerve was in contact with the lingual side of the

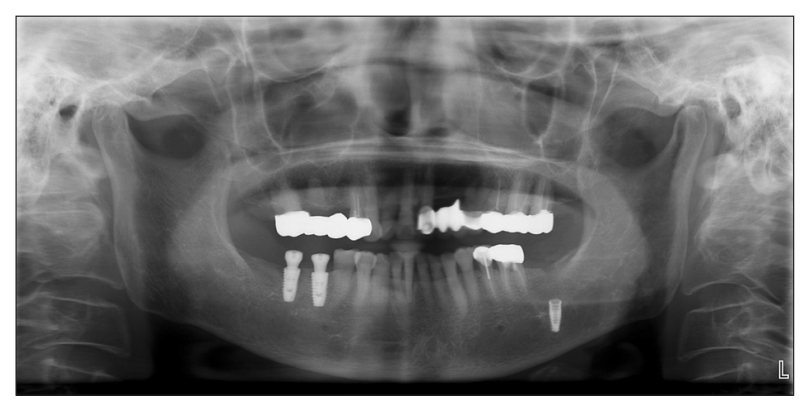

Fig. 1. Implant displaced into the left mandibular bone marrow space in panoramic view. displaced implant (Fig. 2).

Trapezoidal bone window was designed with the inferior base length of $10 \mathrm{~mm}$ and the longitudinal length of $13 \mathrm{~mm}$ with the buccal cortical bone of the drilling hole as the superior margin. Osteotomy was performed on the cortical bone using a Piezosurgery device (Mectron Piezosurgery Device $^{\mathrm{TM}}$; Mectron S.p.a., Carasco Genoa, Italy). After removing the bone window, the surrounding blood clot was carefully removed through saline irrigation and suction to ensure visibility. The implant was gently removed upwards by grasping the exposed upper part without any damage to the mandibular neurovascular bundle. The bone window has been placed in its original location. To prevent nerve injury after surgery, bone graft was not performed. To prevent nerve injury by hematoma or edema, dexamethasone was applied onto the nerve mucoperiosteal flap was replaced and sutured (Fig. 3).

Amoxicillin, prednisolone and nonsteroidal anti-inflammatory drug were prescribed for 1 week after surgery. The sutures were removed 7 days postoperatively. and the healing was uneventful. no neurological complications were noted.

\section{Case 2}

A 48-year-old male with no remarkable medical history

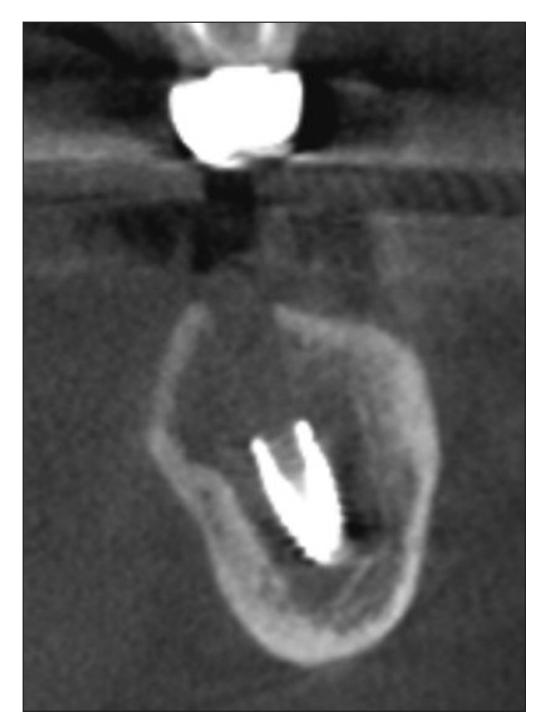

Fig. 2. Implant was located into the bone marrow space and near the inferior alveolar canal. 

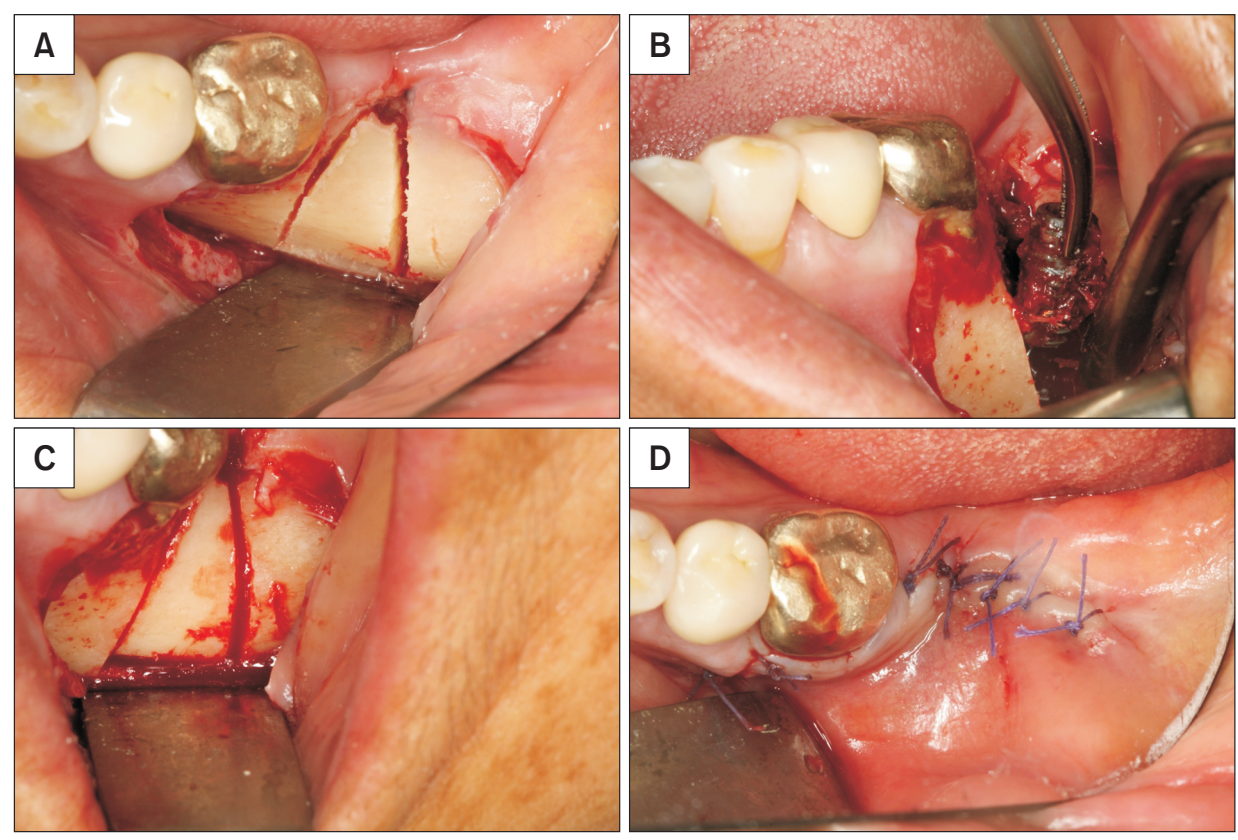

Fig. 3. (A) Trapezoidal osteotomy was performed using a piezoelectric device. (B) The implant was removed. (C) The bony window was replaced in the original position. (D) Wound closure.

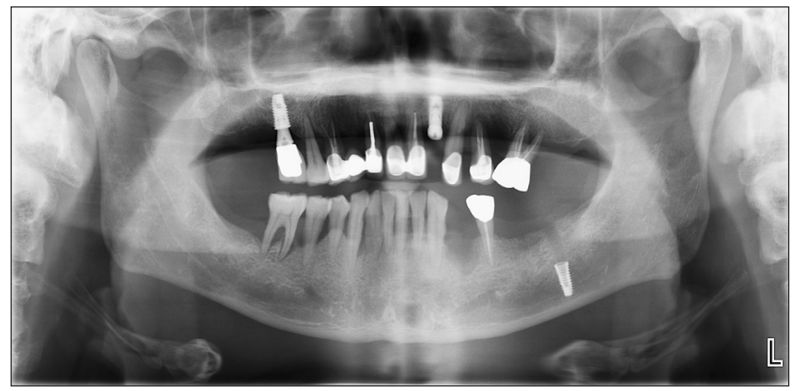

Fig. 4. Implant displaced into the left mandibular bone marrow space near the inferior border in panoramic view.

was referred to our hospital because the implant was displaced downward during implant placement of the mandibular left second molar region. A panoramic radiograph showed that the fixture ( $4.0 \mathrm{~mm}$ wide, $10.0 \mathrm{~mm}$ long) was displaced inferiorly, approximately $10 \mathrm{~mm}$ below the alveolar ridge (Fig. 4). In the $\mathrm{CBCT}$ image, the inferior alveolar nerve was expected to be adjacent just below the displaced implant (Fig. 5). The removal of implant fixture followed the same procedure as that of case 1 . Trapezoidal bone window was designed with the inferior base length of 10 $\mathrm{mm}$ and the longitudinal length of $11 \mathrm{~mm}$ with the buccal cortical bone of the drilling hole as the superior margin (Fig. 6). Three months after surgery the healing was uneventful, and no neurological complications were noted.

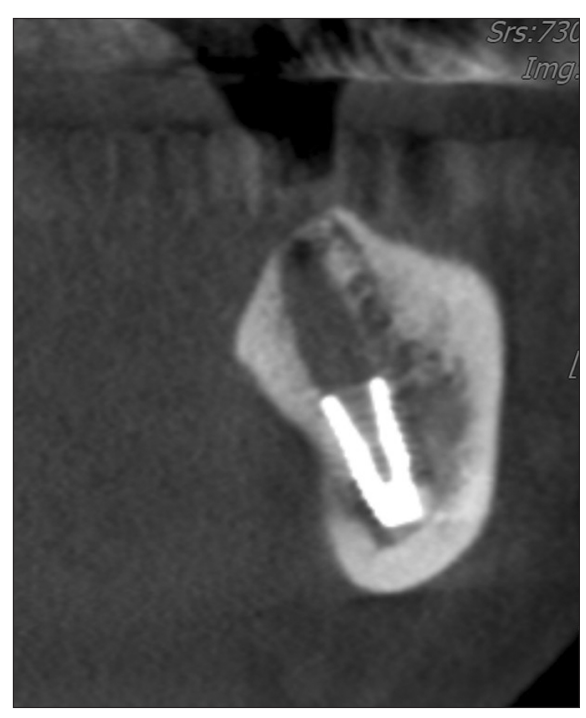

Fig. 5. Implant was displaced into the bone marrow space and closed to the inferior alveolar canal.

\section{DISCUSSION}

Implant displacement into the mandibular bone marrow is mainly due to the focal osteoporotic bone marrow defect [5], which is expected to be associated with women with osteoporosis. However, previous reports suggest that implant displacement could occur in patients without osteoporosis [13]. In addition, there may be an empty space in the bone marrow area for a specific reason such as simple 

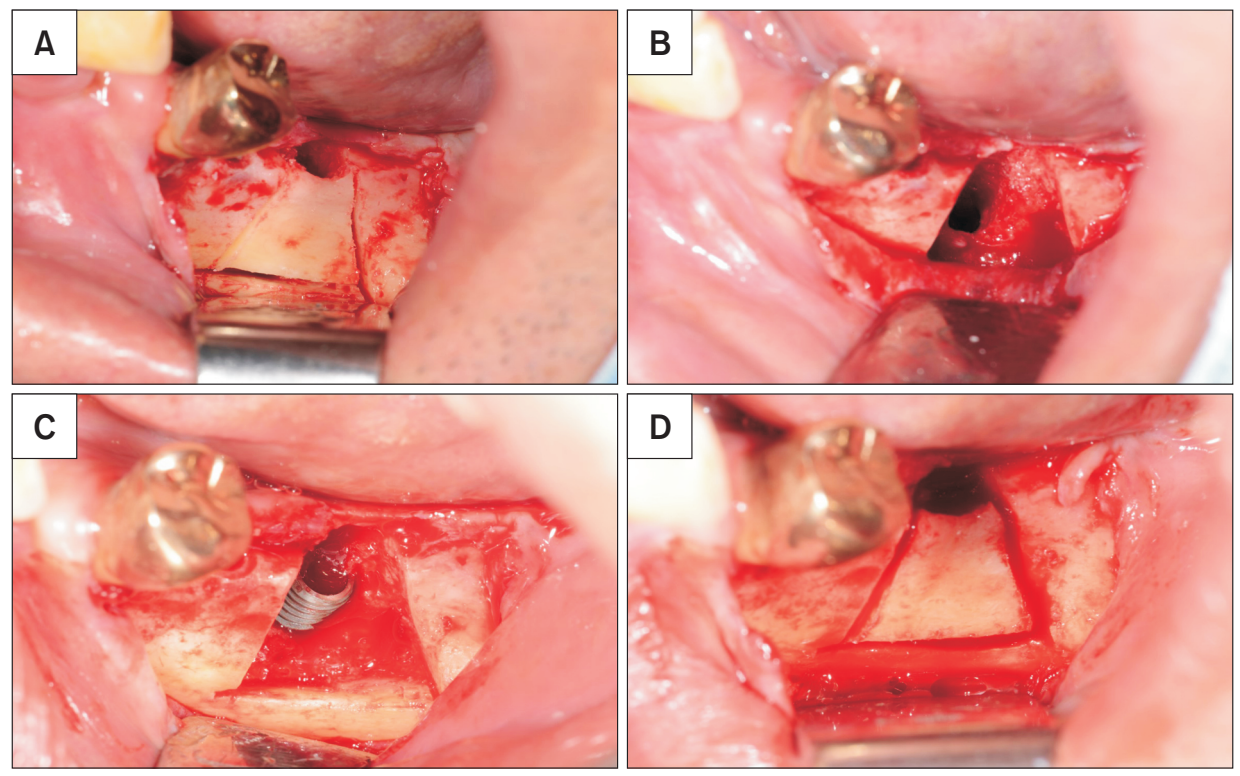

Fig. 6. (A) Trapezoidal osteotomy was performed using a piezoelectric device. (B) Soft tissue-like marrow was observed. (C) The implant was removed. (D) The bony window was replaced in the original position. bone cyst, or there may be a local insufficiency of the trabecular bone with no particular reason [14]. Therefore, the location of implantation and bone density below the location of implantation should be confirmed through preoperative $\mathrm{CBCT}$ images. When the trabecular pattern below the implantation site is not observed on CBCT images, it is necessary to be aware of the displacement of the implant. And in order to maximize the cortical bone engagement, technical procedures such as under-drilling are required.

In most of previously published articles, the implant fixtures displaced into the mandibular bone marrow space were removed in two ways. The first method is to remove it through the crestal approach $[7,9,10]$. This method provides to surgeon limited vision. Also, since the size of the drilling hole in the cortical bone is the same as the diameter of the upper part of the implant fixture, it is not easy to come out and additional bone removal is required. This additional bone removal can be a disadvantage for future implant fixation.

The second method is the lateral approach $[8,12,13]$. This method can secure a better field of view, but the shape of the bony window should be considered. Since the mandibular bone marrow space is not a complete hollow space like the maxillary sinus, the formation of a typical rectangular bony window like the maxillary case may make it difficult to secure sufficient field of view.
The bone window design used in these cases are trapezoidal bone window design, of which upper margin is the buccal area of the implant hole and lower margin is the upper part of the displaced implant. The bone window design would be the most advantageous for minimally invasive implant removal [11]. This is because the bone removal part that was previously used for implant placement can be fully utilized. Since the cortical bone of the buccal side is removed from the alveolar bone, it is advantageous to secure the field of view. Also, since the displaced implant fixture is not fixed in the medullary space, it can be passively removed upwards once the fixture is located. Damage to surrounding anatomical structures, such as the inferior alveolar nerve, will also be least, as removal of the implant fixture does not require rotation or horizontal movement in the medullary space. Finally, trapezoidal bony windows are less likely to move up and down or forward and backward after refitting. Since the upper cortical bone area is included, the bone window itself is less likely to be displaced into the medullary space, thus providing stable healing without fixing through the miniplate.

\section{ACKNOWLEDGEMENTS}

This research was supported by Basic Science Research Program through the National Research Foundation of Ko- 
rea (NRF) funded by the Ministry of Education (2018R1D1A1B07048415).

\section{CONFLICTS OF INTEREST}

The authors declare that they have no competing interests.

\section{ORCID}

\author{
Eun Joo Choi \\ https://orcid.org/0000-0002-6981-8439 \\ Hyo Joon Kim \\ https://orcid.org/0000-0001-6123-4810 \\ Seong Yong Moon \\ https://orcid.org/0000-0002-7513-4404
}

\section{REFERENCES}

1. Brånemark PI. [Jaw reconstruction and intraosseous anchorage of dental prosthesis]. Lakartidningen 1971;68:3105-3116. Swedish.

2. Camargo IB, Van Sickels JE. Surgical complications after implant placement. Dent Clin North Am 2015;59:57-72. doi: 10.1016/j.cden.2014.08.003.

3. Chiapasco M, Felisati G, Maccari A, Borloni R, Gatti F, Di Leo F. The management of complications following displacement of oral implants in the paranasal sinuses: a multicenter clinical report and proposed treatment protocols. Int J Oral Maxillofac Surg 2009;38:1273-1278. doi: 10.1016/j.ijom.2009.09.001.

4. Nakamura N, Mitsuyasu T, Ohishi M. Endoscopic removal of a dental implant displaced into the maxillary sinus: technical note. Int J Oral Maxillofac Surg 2004;33:195-197. doi: 10.1054/ijom.2003.0470.

5. Makek M, Lello GE. Focal osteoporotic bone marrow defects of the jaws. J Oral Maxillofac Surg 1986;44:268-273. doi: 10.1016/0278-2391(86)90077-7.

6. Schneider L, Mesa ML, Fraenkel D. Osteoporotic bone marrow defect: Radiographic features and pathogenic factors. Oral Surg Oral Med Oral Pathol 1988;65:127-129. doi: 10.1016/0030-4220(88)90206-X.

7. Theisen FC, Shultz RE, Elledge DA. Displacement of a root form implant into the mandibular canal. Oral Surg Oral Med Oral Pathol 1990;70:24-28. doi: 10.1016/00304220(90)90172-o.

8. Bayram B, Alaaddinoglu E. Implant-box mandible: dislocation of an implant into the mandible. J Oral Maxillofac Surg 2011;69:498-501. doi: 10.1016/j.joms.2010.10.027.

9. Doh RM, Pang NS, Kim KD, Park W. Implant displacement into the mandible: an unusual complication during implant surgery. Implant Dent 2011;20:345-348. doi: 10.1097/ ID.0b013e3182326813.

10. Lee SC, Jeong CH, Im HY, Kim SY, Ryu JY, Yeom HY, Kim HM. Displacement of dental implants into the focal osteoporotic bone marrow defect: a report of three cases. J Korean Assoc Oral Maxillofac Surg 2013;39:94-99. doi: 10.5125/jkaoms.2013.39.2.94.

11. Kwon YD, Pae A. Piezoelectric trapezoidal osteotomy for the retrieval of a displaced dental implant in the osteoporotic mandibular body. Implant Dent 2016;25:703-706. doi: 10.1097/ID.00000000000000464.

12. Oh JS, Kim SG, You JS. Accidental displacement of the dental implant into the medullary space in the posterior mandible: case reports. J Oral Implantol 2016;42:110-113. doi: 10.1563/aaid-joi-D-13-00349.

13. Kim JW, Paeng JY, Choi SY, Kwon TG. Displacement of dental implants into the mandibular bone marrow space: cause and treatment. a case study and literature review. J Oral Implantol 2017;43:151-157. doi: 10.1563/aaid-joiD-16-00205.

14. Pistilli V, Checchi V, De Paolis F, Pistilli R, Barausse C. Displacement of a dental implant in the mandible body: an unusual case report. Eur J Oral Implantol 2018;11:235240.

15. Scarano A, Lorusso F, Santos de Oliveira P, Murmura G. Dental implants displaced into the mandibular corpus: clinical note. J Dentistry Oral Care 2018;4:8-12. doi: 10.15436/2379-1705.18.1804. 